\title{
Preparation and performance of novel LLTO thin film electrolytes for thin film lithium batteries
}

\author{
CHEN RenJie ${ }^{1,2}$, LIANG Wei ${ }^{1}$, ZHANG HaiQin ${ }^{1}$, WU Feng ${ }^{1,2^{*}} \&$ LI Li $^{1,2}$ \\ ${ }^{1}$ School of Chemical Engineering and Environment, Beijing Key Laboratory of Environmental Science and Engineering, Beijing Institute of \\ Technology, Beijing 100081, China; \\ ${ }^{2}$ National Development Center for High Technology Green Materials, Beijing 100081, China
}

Received November 2, 2011; accepted February 8, 2012; published online July 10, 2012

\begin{abstract}
We prepared a series of lithium lanthanum titanate (LLTO) thin film electrolytes by radio frequency (RF) magnetron sputtering using LLTO targets in a $\mathrm{N}_{2}$ atmosphere. We also deposited the LLTO thin films in an Ar atmosphere under a same condition as references for comparison. The microstructure morphology and the composition of the thin films were investigated by X-ray diffraction (XRD), scanning electron microscopy (SEM) and X-ray photoelectron spectroscopy (XPS), respectively. Results show that the thin film has an amorphous structure with a uniform surface and it is free of pinholes and cracks. Impedance measurements reveal that the ionic conductivity of the electrolytes is beneficial for all solid lithium batteries dependent on the lithium content at room temperature. We found that the amorphous LLTO thin film performs well and it has potential application in microbatteries for use in microelectronic devices.
\end{abstract}

LLTO thin film electrolytes, RF magnetron sputtering, ionic conductivity, lithium batteries

Citation: Chen R J, Liang W, Zhang H Q, et al. Preparation and performance of novel LLTO thin film electrolytes for thin film lithium batteries. Chin Sci Bull, 2012, 57: 4199-4204, doi: 10.1007/s11434-012-5292-y

Thin film energy storage has attracted much attention recently because of the application of ultra-miniaturization in microelectronic devices such as dynamic random access memory (DRAMS) and implantable medical devices [1-3]. By comparison with other microelectronic sources, thin film lithium batteries can potentially store the most energy applied to microelectronic devices because of their high specific energy, long cycle life, good safety performance and environmental friendliness [4-7].

Solid electrolytes are important components in thin film lithium batteries and the performance of a thin film lithium battery is greatly affected by its solid electrolyte [8,9]. It has been reported that the ionic conductivity of $\mathrm{La}_{2 / 3-x} \mathrm{Li}_{3-x}$ $\mathrm{TiO}_{3}(x=0.11)$ is able to reach $10^{-3} \mathrm{~S} / \mathrm{cm}$ at room temperature, making the study of this kind of electrolyte with high ionic conductivity attractive. Recently, lithium lanthanum

*Corresponding author (email: wufeng863@vip.sina.com) titanate (LLTO) has become a very interesting material [10-12] because it has a high rate of ionic conductivity in the amorphous state. And the amorphouse LLTO is one kind of fast ionic conductor [13].

Thin film fabrication can reduce the thickness of the electrolyte layer, resulting in a reduction in the internal resistance of devices. A large surface area is also necessary to achieve a large contact area for a large output current. RF magnetron sputtering is an effective thin film deposition technology for it can be easily used to fabricate large-scale and dense films with outstanding performance [14-16].

In this work, we successfully prepared LLTO thin film electrolytes using RF magnetron sputtering. This constitutes a new chemical composition with an amorphous glassy structure and predominantly ionic conduction. We prepared the nitride LLTO films for the first time with different lithium contents and reported here their effect on structure and ionic conductivity. We also deposited the LLTO thin film in 
an $\mathrm{Ar}$ atmosphere in the same condition for comparison. The properties of all thin films were studied and used as a criterion for finding high performance film electrolytes.

\section{Experimental}

\subsection{Preparation of targets}

The reagents $\mathrm{Li}_{2} \mathrm{CO}_{3}, \mathrm{La}_{2} \mathrm{O}_{3}$ and $\mathrm{TiO}_{2}$ were mixed in different molar ratios $(1.2: 1: 4,1.5: 1: 4,1.8: 1: 4,2: 1: 4)$ and they were melted at $700^{\circ} \mathrm{C}$ for $4 \mathrm{~h}$ in an electrical furnace. Then, the mixtures were calcined at 1050,1100 and $1200^{\circ} \mathrm{C}$, respectively, all for $12 \mathrm{~h}$ [17]. The obtained ceramic was then ball-milled to obtain a fine powder. Finally, the ultima powder compound was pressed into a $60 \mathrm{~mm}$ diameter thin slice. And the slice was sintered at $1100^{\circ} \mathrm{C}$ for $5 \mathrm{~h}$. The composition of the LLTO target was $\mathrm{Li}_{0.6} \mathrm{La}_{0.5} \mathrm{TiO}_{3}, \mathrm{Li}_{0.75^{-}}$ $\mathrm{La}_{0.5} \mathrm{TiO}_{3}, \mathrm{Li}_{0.9} \mathrm{La}_{0.5} \mathrm{TiO}_{3}, \mathrm{LiLa}_{0.5} \mathrm{TiO}_{3}$, respectively.

\subsection{Deposition of the thin films}

The LLTO thin films were deposited on Si-wafer or stainless steel sheet or glass slide which has been spurted two aluminium (Al) layers to make the LLTO flim stuck in the middle, using the target prepared in last step. A working power of $100 \mathrm{~W}$ and a room temperature were applied to the target under the purity $\mathrm{N}_{2}$ atmosphere (purity $99.999 \%$ ). And the gas pressure was kept at $1 \mathrm{~Pa}$ accompanying with the sputtering process lasted for $10 \mathrm{~h}$. Moreover, to guarantee cleanliness, the pressure of the sputtering chamber was lowered to $7.0 \times 10^{-5} \mathrm{~Pa}$ before magnetron sputtering.

The thin film electrolytes were fabricated on a Si-wafer to determine their surface morphology and they were also fabricated on a stainless steel sheet to determine their structures, morphologies and compositions. The sandwich structure which was deposited on glass slide was used to measure the ionic conductivity and electrochemical window of LLTO.

For comparison, non-nitrided LLTO thin film electrolytes were prepared in pure Ar atmosphere under the same conditions.

\subsection{Characterization of properties}

XRD analysis (Rigaku, Japan) was used to determine the crystallinity, crystal parameters and structure of the target and the thin film. The surface morphology was determined using scanning electron microscopy (SEM). And the thickness of the thin film was determined by SEM. The chemical composition of the electrolyte membrane structure was measured using X-ray photoelectron spectroscopy (XPS). Electrochemical impedance spectroscopy and cyclic voltammetry (CV) were carried out using an electrochemical workstation CHI660A in the frequency region from $1 \mathrm{~Hz}$ to $100 \mathrm{kHz}$.

\section{Results and discussion}

\subsection{Morphology and structure of the thin films}

As mentioned above, the electrochemical properties of the thin films are related to their microstructures. The surface roughness of the thin film electrolyte directly affects the ionic conductivity of the electrolyte. A film containing cracks would decrease the battery capacity and could even shorten the cycle life of the battery. The surface and cross-sectional view of the LLTO thin film, which was prepared under an $\mathrm{Ar}$ atmosphere on a Si-wafer substrate, is shown in Figure 1. In Figure 1(a) and (c), the surface of the film is smooth and it has a noncrystalline structure with no cracks or pinholes. Furthermore, the cross-sectional SEM image of the film (Figure 1(c)) shows that the thickness of the film is about $2.63 \mu \mathrm{m}$.

Figure 1(b) shows that the surface of the thin film prepared in a $\mathrm{N}_{2}$ atmosphere is smoother and more uniform with no regular particles or an orderly arrangement by comparing with the film prepared under an $\mathrm{Ar}$ atmosphere. The thickness of the film that was prepared under a $\mathrm{N}_{2}$ atmosphere is about $1.5 \mu \mathrm{m}$ less than the film prepared under an Ar atmosphere (Figure 1(d)). The results indicate that the choice of synthesis atmosphere is important because it greatly influences the microstructure of the films.

Figure 2(a) shows the XRD pattern of the LLTO target in which the lithium content is $150 \%$. The LLTO target has some sharp diffraction peaks, and these are due to the diffraction of crystalline LLTO. Figure 2(b) shows the XRD pattern of LLTO film. The microcrystalline peaks respectively correspond with the peak of (101) facet $\left(25^{\circ}\right)$ and (110) facet $\left(35^{\circ}\right)$ of LLTO target observed in Figure 1(a). There are no sharp diffraction peaks which are diffracted by crystals in the XRD pattern of LLTO film. As expected, the LLTO thin film electrolyte that was deposited under a $\mathrm{N}_{2}$ atmosphere onto the stainless steel substrate using the above mentioned target has an amorphous structure. The XRD pattern for the thin film electrolyte only shows sharp peaks from the stainless steel substrate (i.e. $43.582^{\circ}, 50.791^{\circ}$ and $\left.74.697^{\circ}\right)$

Therefore, an amorphous LLTO thin film was obtained when using the crystalline LLTO target and the RF magnetron sputtering method at a $\mathrm{N}_{2}$ working pressure of $1 \mathrm{~Pa}$, at room temperature and with an RF power of $100 \mathrm{~W}$.

XPS was used to characterize the chemical nature of thin film electrolytes. Figure 3(a) shows the XPS spectra of the nitride LLTO thin film with the lithium content of $150 \%$. The XPS spectra consist of peaks assigned to $\mathrm{Li}, \mathrm{La}, \mathrm{Ti}, \mathrm{O}$, $\mathrm{N}$ and C. C1s was used as an internal reference $(284.8 \mathrm{eV})$. An obvious peak around $396.0 \mathrm{eV}$ was observed and this is attributed to the nitrogen incorporation. The composition of the thin films can be calculated using the following equation: 


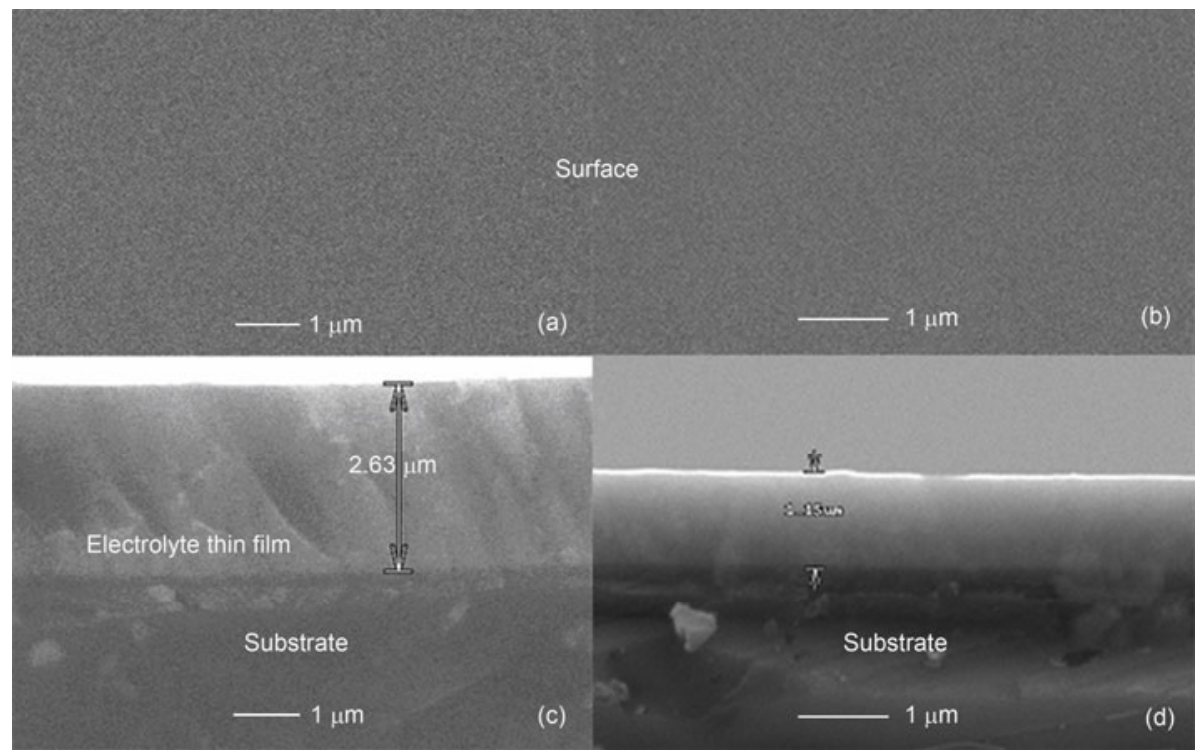

Figure 1 SEM images of the surface (a) and cross-section (c) of the LLTO (lithium content is 150\%) thin film prepared under an Ar atmosphere, and the surface (b) and cross-section (d) of the LLTO thin film prepared under a $\mathrm{N}_{2}$ atmosphere.

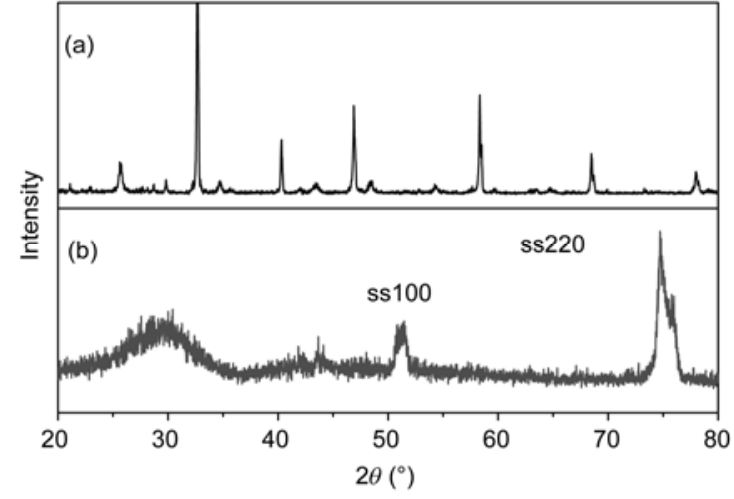

Figure 2 XRD patterns of the LLTO $\left(\mathrm{Li}_{0.75} \mathrm{La}_{0.5} \mathrm{TiO}_{3}\right)$ target (a) and the LLTO $\left(\mathrm{Li}_{0.75} \mathrm{La}_{0.5} \mathrm{TiO}_{3}\right)$ thin film (b) prepared under a $\mathrm{N}_{2}$ atmosphere.

$$
C_{x}=\frac{n_{x}}{\sum_{i=1}^{k} n_{i}}=\frac{I_{x} / S_{x}}{\sum_{i=1}^{k} I_{i} / S_{i}},
$$

where $n_{x}$ is the atomic number density, $k$ is the number of elements detected in the sample, $I_{x}$ is the photoelectron emission intensity of a specific element $x$ in the XPS survey spectrum and $S_{x}$ is the atomic sensitivity factor of element $x$ in the sample, as determined from the empirical peak area data [18].

Figure 3(b) illustrates the N 1s XPS spectrum of the thin film. It indicates that the nitrogen is incorporated into the internal structure of the film. There are two asymmetric peaks at 395.4 and $399.8 \mathrm{eV}$ and they can be attributed to two different bonding states of nitrogen atoms [19]. The peak at $395.4 \mathrm{eV}$ represents nitrogen bonds of doubly coordinated nitrogen $-\mathrm{N}=$ and the other feeblish peak represents
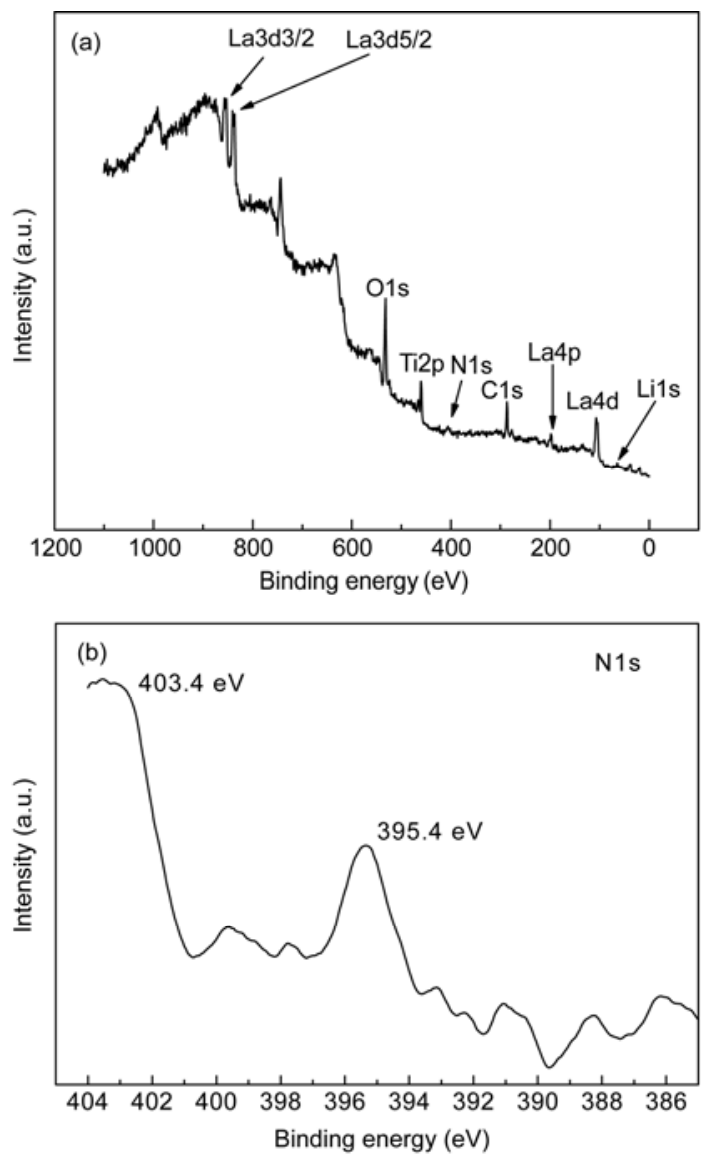

Figure 3 XPS of the LLTO thin firm (a) and the N1s region of the LLTO thin film (b) prepared under a $\mathrm{N}_{2}$ atmosphere.

triply coordinated nitrogen $-\mathrm{N}<$. This indicates that the nitrogen actually substitutes the partial oxygen during deposition. Hence, $-\mathrm{N}=$ and $-\mathrm{N}<$ nitrogen atoms replace $-\mathrm{O}-$ and 
$=\mathrm{O}$ oxygen atoms making for a more cross-linking network in internal structure of the film. This micro-structural modification was considered to be beneficial for the ionic conduction and electrochemical stability of film electrolytes.

\subsection{Analysis of impedance properties}

Ionic conductivity is a very important parameter for thin film electrolytes and the ionic conductivity of the LLTO films was obtained using the following equation:

$$
\sigma=d /(R \cdot A),
$$

where $d$ is the thickness of the film, $A$ is SS electrode/electrolyte interface area and $R$ is the resistance of the film that is determined from the measured impedance by selecting the value of $Z^{\prime}$ when $-Z^{\prime \prime}$ goes through a local minimum in the electrochemical impedance spectra [20,21].

Impedance spectroscopy was carried out to determine the ionic conductivity using a sandwich structure blocking electrode cell (Al/LLTO/Al). The impedance spectra are typical of dielectric relaxation processes. The impedance spectra of the LLTO thin film electrolytes prepared under $\mathrm{N}_{2}$ and $\mathrm{Ar}$ atmospheres are shown in Figures 4 and 5, respectively. It seems that the spectra are normal alternating current (AC) impedance spectra of electronic conductors with no straight sloping line at the low frequency end.

Figure 4 shows the impedance spectra of the LLTO thin film electrolytes with different lithium content which were prepared under a $\mathrm{N}_{2}$ atmosphere. We found that an optimal lithium content exists and the highest ionic conductivity was achieved for a lithium content of $150 \%$.

From Figure 5(a) it is obvious that the LLTO thin film electrolyte that was prepared under an Ar atmosphere is an electronic conductor at lithium content lower than $150 \%$, which is not suitable for solid-state thin film battery electrolytes. However, at lithium content of $150 \%$ the ionic conductivity of the thin film electrolyte can reach $10^{-6} \mathrm{~S} / \mathrm{cm}$, which is very similar to that reported in the literature [17]. At a lithium content of $180 \%$ the ionic conductivity decreased to $3 \times 10^{-7} \mathrm{~S} / \mathrm{cm}$. At a lithium content of $200 \%$ the battery was shortened. It is obvious from Figure 5(b) that the surface of this film has defects such as cracks and pinholes,

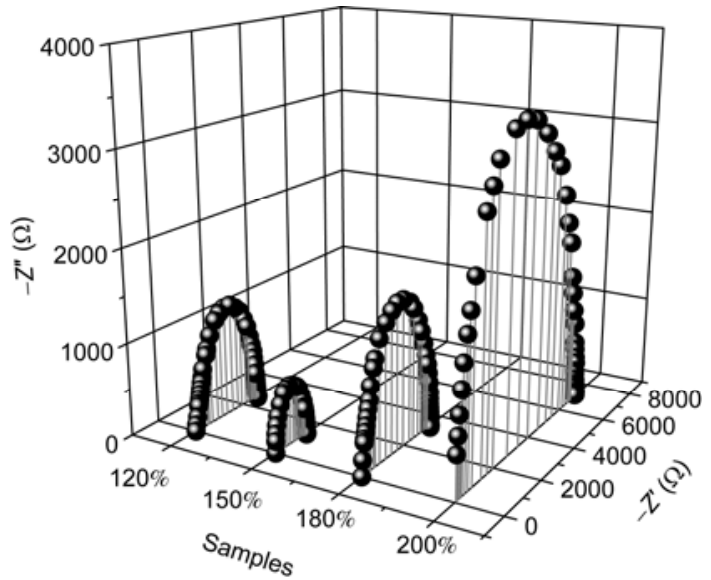

Figure 4 Electrochemical impedance spectroscopy of the LLTO thin films with different lithium content prepared under a $\mathrm{N}_{2}$ atmosphere.
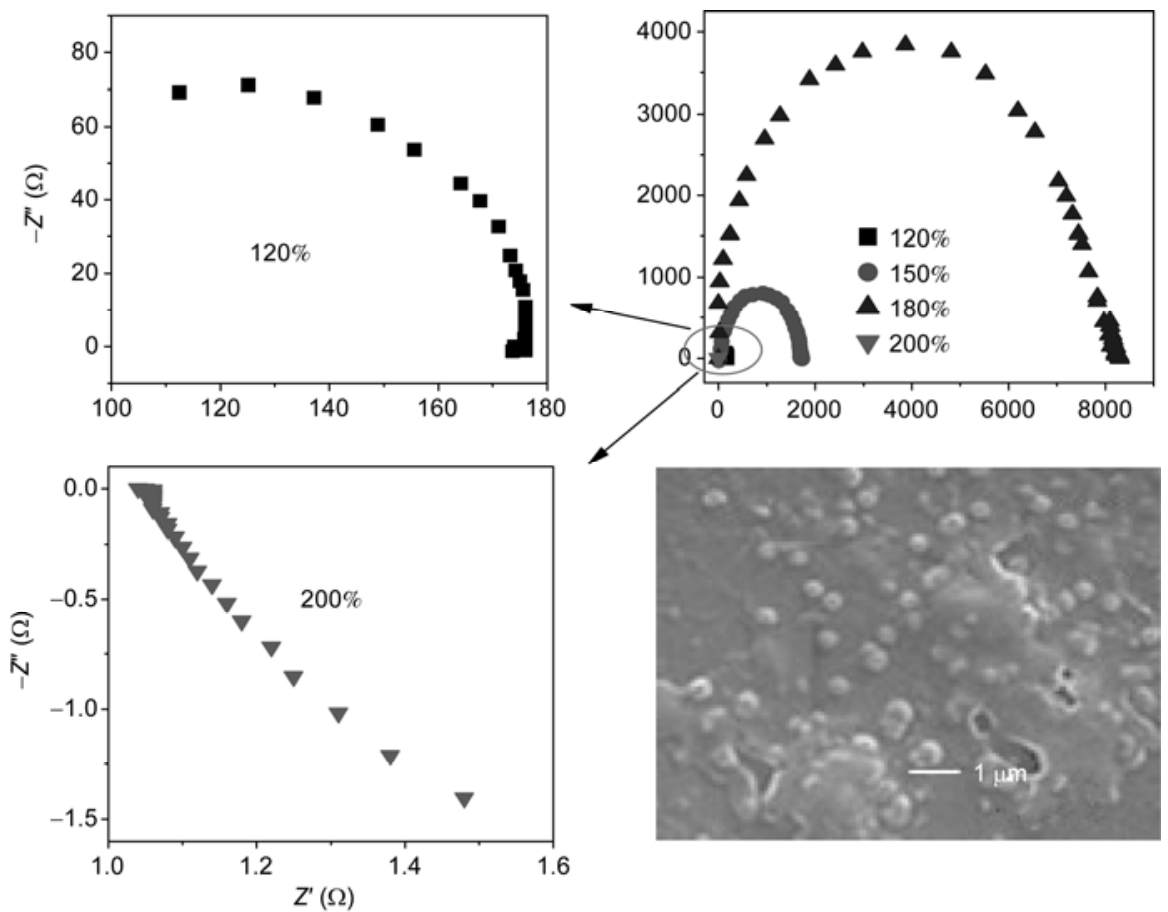

Figure 5 Electrochemical impedance spectroscopy of the LLTO thin films with different lithium contents prepared under an Ar atmosphere and a SEM image of the surface of the LLTO thin film for a lithium content of $200 \%$ prepared under an Ar atmosphere. 
which results in its poor electrochemical performance. Comparing with the LLTO films fabricated under an $\mathrm{Ar}$ atmosphere, the LLTO films fabricated under a $\mathrm{N}_{2}$ atmosphere show the nature of the ion conductor in a more wider range of lithium content. The nitride LLTO films have the better combination property than no-nitride LLTO films with an approximate ionic conductivity.

From above results, the LLTO films with $150 \%$ lithium content deposited in $\mathrm{N}_{2}$ or $\mathrm{Ar}$ atmosphere both reveal the biggest ionic conductivity. According to the equation, $\sigma=$ charge density $\times$ mobility, the ionic conductivity is determined by the product of charge density and mobility [22]. Therefore, there are two ways to increase the $\mathrm{Li}^{+}$ionic conductivity of thin films. One is increasing the $\mathrm{Li}^{+}$content to increase the charge carrier density. Another reason for the increase in ionic conductivity is related to the ionic mobility. Especially, in nitride films, $\mathrm{N}^{3-}$ substitution of $\mathrm{O}^{2-}$ leads to a more reticulated network for the faster ionic mobility. These cross-linking structures create more conduction paths with a lower activation energy and give rise to a notable modification of glass properties. However, when the lithium content overtops the ratio of $150 \%$, the ionic conductivity decreases obviously, and this is due to the ions gather which prevent ionic conduction resulting low charge transport on electrode-electrolyte interface.

Electrochemical stability is also an essential criterion for a solid electrolyte. Figure 6 shows the cyclic voltammogram of the nitride LLTO thin film with lithium content of $150 \%$. The irreversible onset of the current can be defined as the electrolyte breakdown voltage. An obvious current charge occurred above $2.0 \mathrm{~V}$ at high voltage and another obvious current charge occurred at $-1.5 \mathrm{~V}$ at low voltage, this suggests that the film remains stable at high potentials of more than $3.0 \mathrm{~V}$ where lithium batteries usually operate. This thin film electrolyte is, therefore, predicted to be a promising candidate for all-solid-state thin film lithium batteries [23]. Another nitride LLTO film with lithium content of $120 \%$

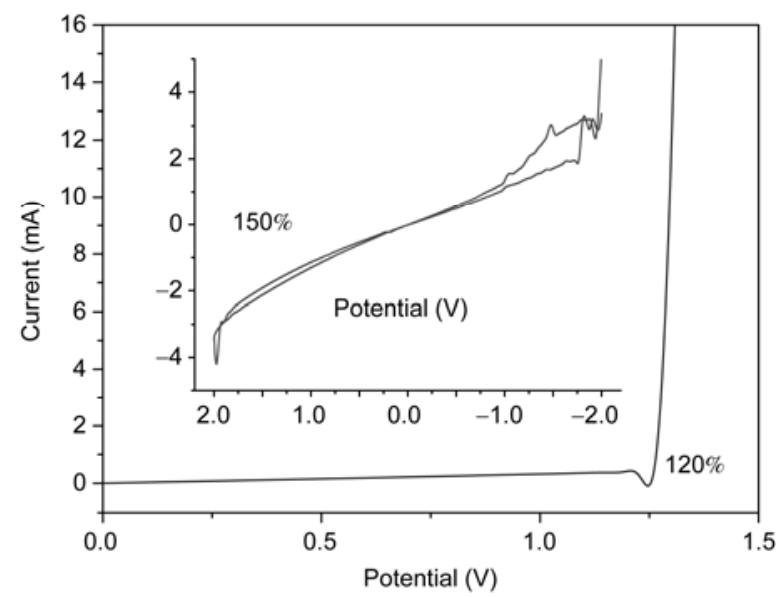

Figure 6 Cyclic voltammogram of the LLTO thin film prepared under a $\mathrm{N}_{2}$ atmosphere with lithium contents of $120 \%$ and $150 \%$. was used as a comparison, which shows a lower decomposition voltage at $1.2 \mathrm{~V}$ at high voltage and its electrochemical stability is worse than the film with lithium content of $150 \%$.

\section{Conclusion}

A series of LLTO thin film electrolytes were prepared by RF magnetron sputtering using LLTO targets under a pure $\mathrm{N}_{2}$ atmosphere. Their morphological structures and electrochemical properties were investigated in detail. The results show that the films possess amorphous structures and are smooth, dense and uniform with no cracks or pinholes. Furthermore, the ionic conductivity of the films that were prepared under a $\mathrm{N}_{2}$ atmosphere can reach $9.4 \times 10^{-7} \mathrm{~S} / \mathrm{cm}$ at room temperature when the lithium content is $150 \%$. These kinds of electrolytes are close to the film deposited in an $\mathrm{Ar}$ atmosphere in morphology and performance. The serious electrolytes have good properties and are promising candidate materials for all solid state thin film lithium batteries.

This work was supported by the National Basic Research Program of China (2009CB220100), the Key International S\&T Cooperation Projects of China (2010DFB63370), the National High-tech R\&D Program of China (2011AA11A256), New Century Educational Talents Plan of Chinese Ministry of Education (NCET-10-0038) and Beijing Novel Program (2010B018).

$1 \mathrm{Hu} \mathrm{Z} \mathrm{Q,} \mathrm{Xie} \mathrm{K,} \mathrm{Wei} \mathrm{D,} \mathrm{et} \mathrm{al.} \mathrm{Influence} \mathrm{of} \mathrm{sputtering} \mathrm{pressure} \mathrm{on} \mathrm{the}$ structure and ionic conductivity of thin film amorphous electrolyte. J Mater Sci, 2011, 46: 9588-9593

2 Yada C, Iriyama Y, Abe T, et al. A novel all-solid-state thin-filmtype lithium-ion battery with in situ prepared positive and negative electrode materials. Electrochem Commun, 2009, 11: 413-416

3 Kim S, Dulin A, Shenoy V. Effect of electrolytes on the structure and evolution of the solid electrolyte interphase (SEI) in Li-ion batteries: A molecular dynamics study. J Power Sources, 2011, 196: 8590-8597

4 Jee S H, Lee M J, Ahn H S, et al. Characteristics of a new type of solid-state electrolyte with a LiPON interlayer for Li-ion thin film batteries. Solid State Ionics, 2010, 181: 902-906

5 Ho W H, Li C F, Liu H C, et al. Electrochemical performance of $\operatorname{In}_{2} \mathrm{O}_{3}$ thin film electrode in lithium cell. J Power Sources, 2008, 175: 897-902

6 Lee Y G, Kim J, Kim S, et al. 3.0 V-class film-type lithium primary battery with highly improved energy density. J Power Sources, 2010, 195: 3715-3719

7 Hayashi M, Takahashi M, Shodai T. Preparation and electrochemical properties of pure lithium cobalt oxide films by electron cyclotron resonance sputtering. J Power Sources, 2009, 189: 416-422

8 Li C L, Zhang B, Fu Z W. Physical and electrochemical characterization of amorphous lithium lanthanum titanate solid electrolyte thin-film fabricated by e-beam evaporation. Thin Solid Films, 2006, 515: 1886-1892

9 Nougaret L, Combette P, Pascal-Delannoy F. Growth of lithium tantalate thin films by radio-frequency magnetron sputtering with lithium enriched target. Thin Solid Films, 2009, 517: 1784-1789

10 Li C L, Fu Z W. All-solid-state rechargeable thin film lithium batteries with $\mathrm{Li}_{x} \mathrm{Mn}_{2} \mathrm{O}_{4}$ and $\mathrm{Li} x \mathrm{Mn} 2 \mathrm{O} 4-0.5 \mathrm{ZrO}(2)$ cathodes. Electrochim Acta, 2007, 52: 6155-6164

11 Xie J, Imanishi N, Zhang T, et al. Amorphous LiCoO(2) thin films on $\mathrm{Li}(1+x+y) \operatorname{Al}(x) \operatorname{Ti}(2-x) \operatorname{Si}(y) \mathrm{P}(3-y) \mathrm{O}(12)$ prepared by radio frequency magnetron sputtering for all-solid-state Li-ion batteries. Electrochim Acta, 2010, 55: 5440-5445 
12 Šalkus T, Kazakevičius E, Kežionis A, et al. Determination of the non-Arrhenius behaviour of the bulk conductivity of fast ionic conductors LLTO at high temperature. Solid State Ionics, 2011, 188: 69-72

13 Gong Z Q, Wang Z X. Modern Electrochemistry. Changsha: Central South University Press, 2010

$14 \mathrm{Xu} \mathrm{L}$, Wang R, Liu Y, et al. Influence of post-annealing on the properties of Ta-doped $\mathrm{In}_{2} \mathrm{O}_{3}$ transparent conductive films. Chin Sci Bull, 2011, 56: 1535-1538

15 Sasaki K, Muranaka M, Suzuki A, et al. Synthesis and characterization of LSGM thin film electrolyte by RF magnetron sputtering for LT-SOFCS. Solid State Ionics, 2008, 179: 1268-1272

16 Kuo Y L, Lee C, Chen Y S, et al. Gadolinia-doped ceria films deposited by RF reactive magnetron sputtering. Solid State Ionics, 2009, 180: $1421-1428$

17 Xiong Y L, Tao H Z, Zhao J, et al. Effects of annealing temperature on structure and opt-electric properties of ion-conducting LLTO thin films prepared by RF magnetron sputtering. J Alloy Compd, 2011,
509: 1910-1914

18 Wan D, Komvopoulos K. Tetrahedral and trigonal carbon atom hybridization in thin amorphous carbon films synthesized by radio-frequency sputtering. J Phys Chem C, 2007, 111: 9891-9896

19 Fleutot B, Pecquenard B, Martinez H, et al. Investigation of the local structure of LiPON thin films to better understand the role of nitrogen on their performance. Solid State Ionics, 2011, 186: 29-36

20 Wu F, Liu Y D, Chen R J, et al. Preparation and performance of novel Li-Ti-Si-P-O-N thin-film electrolyte for thin-film lithium batteries. J Power Sources, 2009, 189: 467-470

21 Kim J M, Park G B, Lee K C, et al. Li-B-O-N electrolytes for allsolid-state thin film batteries. J Power Sources, 2009, 189: 211-216

22 Roh N S, Lee S D, Kwon H S. Effects of deposition condition on the ionicconductivity and structure of amorphous lithium phosphorus oxynitrate thin film. Scripta Mater, 2000, 42: 43-49

23 Zhao S L, Qin Q Z. Li-V-Si-O thin film electrolyte for all-solid-state Li-ion battery. J Power Sources, 2003, 122: 174-180

Open Access This article is distributed under the terms of the Creative Commons Attribution License which permits any use, distribution, and reproduction in any medium, provided the original author(s) and source are credited. 